\title{
Possible Pathogenetic Relationship between Fabry Disease and Renal Cell Carcinoma
}

\author{
Fabio Pagni $^{\mathrm{a}}$ Federico Pieruzzi $^{\mathrm{b}}$ Stefano Zannella ${ }^{\mathrm{a}}$ Antonella Di Giacomo ${ }^{\mathrm{b}}$ \\ Giorgio Bovo $^{a}$ Franco Ferrario $^{a} \quad$ Giacomo Torti $^{b}$ Rodolfo Rivera $^{b}$ Emma Assi $^{c}$ \\ Federica Viglione $^{e}$ Manuela Nebuloni ${ }^{d}$ \\ Departments of a Pathology and ${ }^{\mathrm{b}}$ Nephrology, San Gerardo Hospital, University of Milan-Bicocca, Monza, \\ Departments of ${ }^{\mathrm{C} P h a r m a c o l o g y}$ and ${ }^{\mathrm{d}}$ Pathology, Sacco Hospital, University of Milan, Milan, and ${ }^{\text {L Laboratory of }}$ \\ Histology, Institute of Clinical Physiology, CNR, Pisa, Italy
}

\section{Key Words}

Fabry disease $\cdot$ Renal cell carcinoma $\cdot$ Zebra bodies $\cdot$ Renal biopsy

\begin{abstract}
The occurrence of renal cell carcinoma (RCC) in Fabry disease (FD) is a rare event. We report a deep ultrastructural study of RCC in a patient with a previous histological diagnosis of FD. In order to highlight analogies and differences between the two histological samples, we used the nephrectomy specimen as a 'repeat biopsy', making a dynamic analysis of the evolution of the disease-related kidney damage. Secondly, a comparative ultrastructural analysis between non-neoplastic tissue and cancer demonstrated for the first time the presence of zebra bodies in the tumor cells. Finally, a hypothetical speculation about the relationship between the lysosomal accumulation, the oxidative damage and the genesis of the tumor was performed. The link connected the accumulation of glycosphingolipid globotriaosylceramide, characteristic of FD, with the expression of CD74 and macrophage migration inhibitory factor that may play an important role in tumorigenesis regulated by the Von Hippel-Lindau/hypoxia-inducible factor $1 \alpha$ pathway.

Copyright $\odot 2012$ S. Karger AG, Basel
\end{abstract}

\section{KARGER}

Fax +41613061234 E-Mail karger@karger.ch www.karger.com

\section{() 2012 S. Karger AG, Basel \\ 0250-8095/12/0366-0537\$38.00/0}

Accessible online at:

www.karger.com/ajn

\section{Introduction}

Fabry disease (FD) is an X-linked syndrome caused by the missing activity of the enzyme $\alpha$-galactosidase $\mathrm{A}$ which results in a progressive and systemic accumulation of glycosphingolipid globotriaosylceramide (Gb3). In most cases, the advanced disease leads to a progressive deterioration of kidney function due to the presence of typical deposits in renal cells, especially podocytes. These deposits, which are called myelin bodies or zebra bodies, are best shown thanks to transmission electron microscopy (TEM) and could have different sizes and shapes. The occurrence of renal cell carcinoma (RCC) in FD is an rare event. Only two other cases of (bilateral) RCC in a genetically confirmed FD have been reported in the literature $[1,2]$. No etiologic correlation between FD and tumor development was demonstrated. Moreover, these tumors did not present peculiar features compared with conventional (sporadic and hereditary) forms.

\section{F. Pagni and F. Pieruzzi contributed equally to this paper.}

Dr. Fabio Pagni

Department of Pathology, San Gerardo Hospital, University of Milan-Bicocca

IT- 20900 Monza (Italy)

E-Mail fabio.pagni@unimib.it 

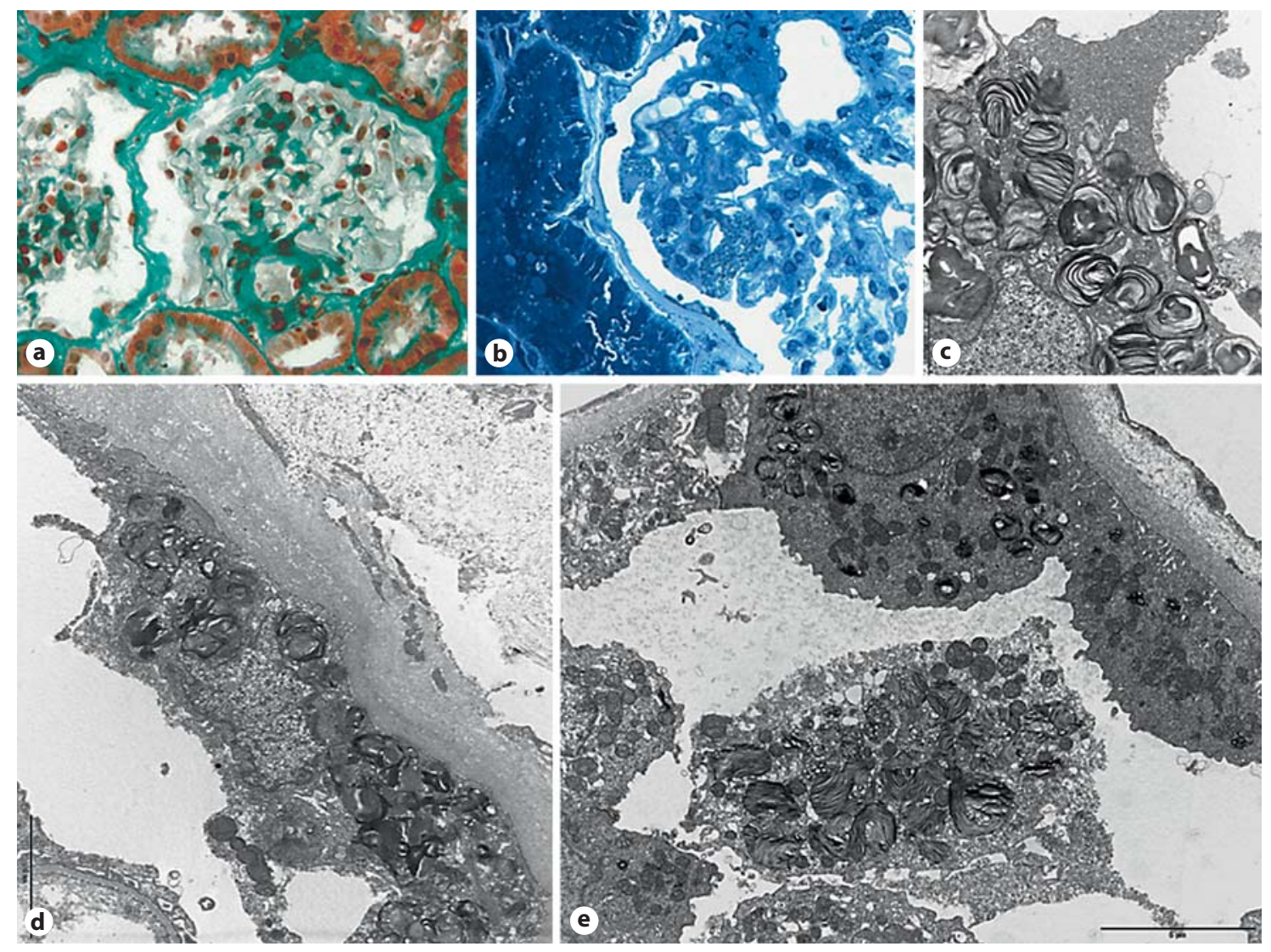

Fig. 1. Renal biopsy. a Initial segmental sclerosis was evident in some glomeruli; swelling podocytes with vacuolated cytoplasm were occasionally found. Masson trichrome. $\times 40$. b High-power field histology revealed some diagnostic, typical inclusions in glomeruli. Toluidine blue. $\times 100$. c-e Gb3-rich lipidic lysosomal inclusions in podocytes, parietal cells and distal tubular cells. TEM. $\times 300$.

\section{Case Report}

After a diagnosis of FD in her third son, a 45-year-old Peruvian female patient was found to have proteinuria $(286 \mathrm{mg} / 24 \mathrm{~h}$, albuminuria $154 \mathrm{mg} / 24 \mathrm{~h}$, creatinine clearance $160 \mathrm{ml} / \mathrm{min}$ ) associated with neurosensorial hearing loss, corneal whirling and paresthesias. $\alpha$-Galactosidase A enzyme activity in plasma leukocytes was low (26 nmol/h/mg; normal range: $30.2-107.5)$. The patient underwent renal biopsy, which suggested an FD diagnosis (fig. 1). A genetic analysis (polymerase chain reaction and direct DNA sequencing) found a new mutation both in her son's skin fibroblasts and her peripheral blood. This spot mutation (heterozygosis, c. $4 \mathrm{C}>\mathrm{T}$ ) causes a Gln2Stop non-sense mutation. Sequencing analysis of exon 1 showed presence of the polymorphism g.1170C $>$ T [3]. Angiotensin-converting enzyme inhibitor and enzyme replacement therapy (ERT) was planned, but the patient did not optimally comply with the treatment during the follow-up. However, she underwent annual ultrasound examination. Six years later, a hyperechogenic nodule in the right kidney appeared. Computerized tomography showed a solid and exophytic lesion in the middle-upper pole of the kidney (fig. 2a). There was no clinical evidence of renal failure. The patient underwent a nephrectomy, and the lesion was diagnosed as an RCC. After the surgery, we observed a progressive worsening of creatinine clearance $(55 \mathrm{ml} / \mathrm{min})$, and a tendency to higher pressure levels. For these reasons, angiotensin-converting enzyme inhibitor therapy was confirmed, with a strong recommendation to start ERT.

\section{Renal Biopsy Findings}

For the histological analysis, a $1.5-\mathrm{cm}$-long renal core biopsy was fixed with $10 \%$ buffered formalin and afterward paraffin embedded. $3-\mu$ m-thick sections were cut from the block and set for hematoxylin-eosin and histochemical stains. A little fragment of the biopsy was frozen and sent for immunofluorescence (IF). A third little sample was taken for TEM, fixed in glutaraldehyde, dehydrated and embedded in Epon. Resin-embedded sections were stained with toluidine blue. Histological sections revealed the presence of 15 glomeruli with absence of mesangial alterations and normal basal membrane (fig. 1a); 2 out of 15 glomeruli were totally sclerotic. Segmental sclerosis was focally present (fig. 1a). Podocytes showed mild bulging and vacuolated aspects (fig. 1a). Some tubular cells with foamy cytoplasm were found. At high- 

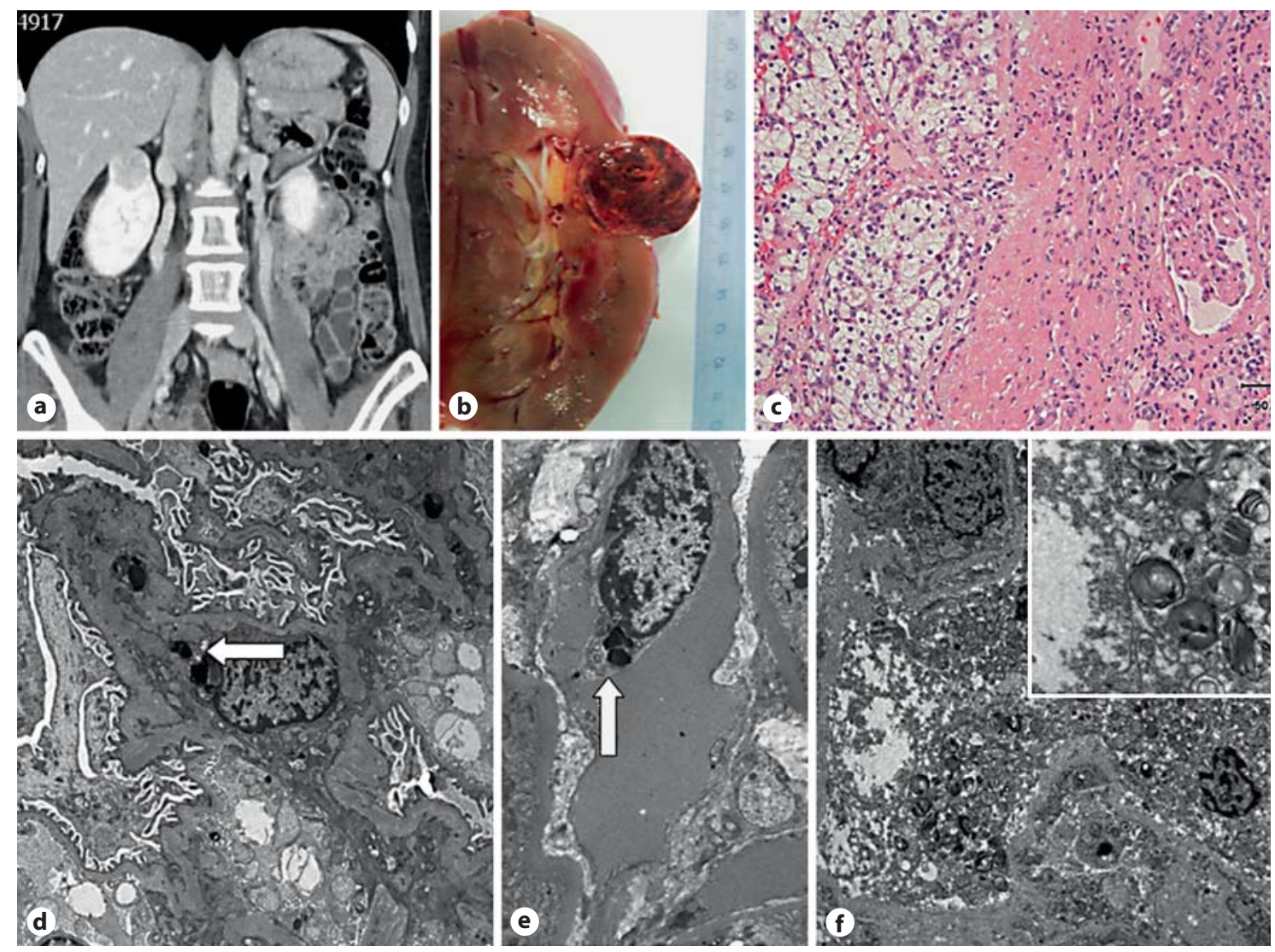

Fig. 2. Nephrectomy. a A nodular mass was evident in the upper pole of the kidney (computerized tomography imaging). b The macroscopic specimen showed a yellow mass of the right kidney with an expansive growth pattern. c Segmental sclerosis in cortical glomeruli near RCC cells. HE. $\times 10$. d Tiny Gb3 inclusions in mesangial cell (arrow). TEM. $\times 300$. e Gb3 inclusions in endothe-

lial cell (arrow). TEM. $\times 300 . \mathbf{f}$ Gb3 lysosomal inclusions in tumor cells. Tumor cell inclusions are similar to those observed in epithelial cells. On the left of the image, granular inclusions and electron-lucent areas are seen suggestive of glycogen in partial dissolution, consistent with RCC. In the inset, typical FD lamellar inclusions are visible. TEM. $\times 300$; inset $\times 700$.

power field, toluidine blue staining revealed inclusions suspicious of zebra bodies (fig. 1b). IF (IgG, IgA, IgM, C3, C1q, fibrinogen, kappa and lambda immunoglobulin light chains) resulted completely negative. TEM showed rarefaction and vacuolization of visceral epithelium and endothelial cells, with irregularly thick basal membrane and frequent thin shares. Zebra bodies with cytoplasmic localization were found with a focal pattern of distribution in visceral (fig. 1c), parietal (fig. 1d) and tubular (fig. 1e) cells. No inclusion in endothelial cells was present.

\section{Nephrectomy Findings}

A $10 \times 4.5 \times 3 \mathrm{~cm}$ right nephrectomy specimen was submitted for histological analysis. The kidney showed a $2.7-\mathrm{cm}$ maximum diameter nodular mass with yellow cut surface located in the central region (fig. 2b). Histologically, the tumor was constituted by clear cells with classic features of conventional RCC (Fuhrman grade 2; fig. 2c). The peritumoral tissue showed some totally sclerotic glomeruli; segmental sclerosis with visceral epithelium hypertrophy was appreciable with a more diffuse pattern

than in renal biopsy. Cytoplasmic vacuolization of the tubular epithelium and minimal peritubular and vascular fibrosis were reported. IF was completely negative again. EM showed Gb3-rich lipidic lysosomal inclusions and lamellar structures in podocytes, parietal and tubular cells. Myelin-like structures and tiny Gb3 inclusions were evident also in mesangial cells (fig. $2 \mathrm{~d}$ ). In contrast to the first biopsy, the Gb3 inclusions were present also in the endothelial cells of the glomerular and interstitial vessels (fig. 2e). TEM revealed Gb3 lysosomal inclusions in tumor clear cells (fig. 2f) and endothelial cells. Finally, the nephrectomy specimen was stained with CD74 (immunohistochemistry LN2 at 1:50 dilution, Dako, Glostrup, Denmark) and macrophage migration inhibitory factor (MIF sc_20121 at 1:100 dilution, Santa Cruz Biotechnology, Santa Cruz, Calif., USA). CD74 (fig. 3a) and its ligand MIF (fig. 3b) displayed a prominent membrane localization at immunohistochemistry in tumor cells, as previously described [4]. Moreover, patchy reactivity was also evident in glomeruli and tubules (fig. 3c, d). 
Fig. 3. CD74 and MIF expression in the nephrectomy specimen. a Strong cytoplasmic and membrane reactivity of CD74LN2 in clear cells. Immunohistochemistry. $\times 20$. b MIF expression in clear cells. Immunohistochemistry. $\times 20$ and $\times 40$. Note the prominent membranous pattern of reactivity. c Patchy reactivity in the glomerulus. Both CD74 and MIF had the same pattern. Immunohistochemistry. $\times 40$. d Tubular expression (note the atypical cytoplasm signal and the apical, membranous true reinforcement). Immunohistochemistry. $\times 40$.
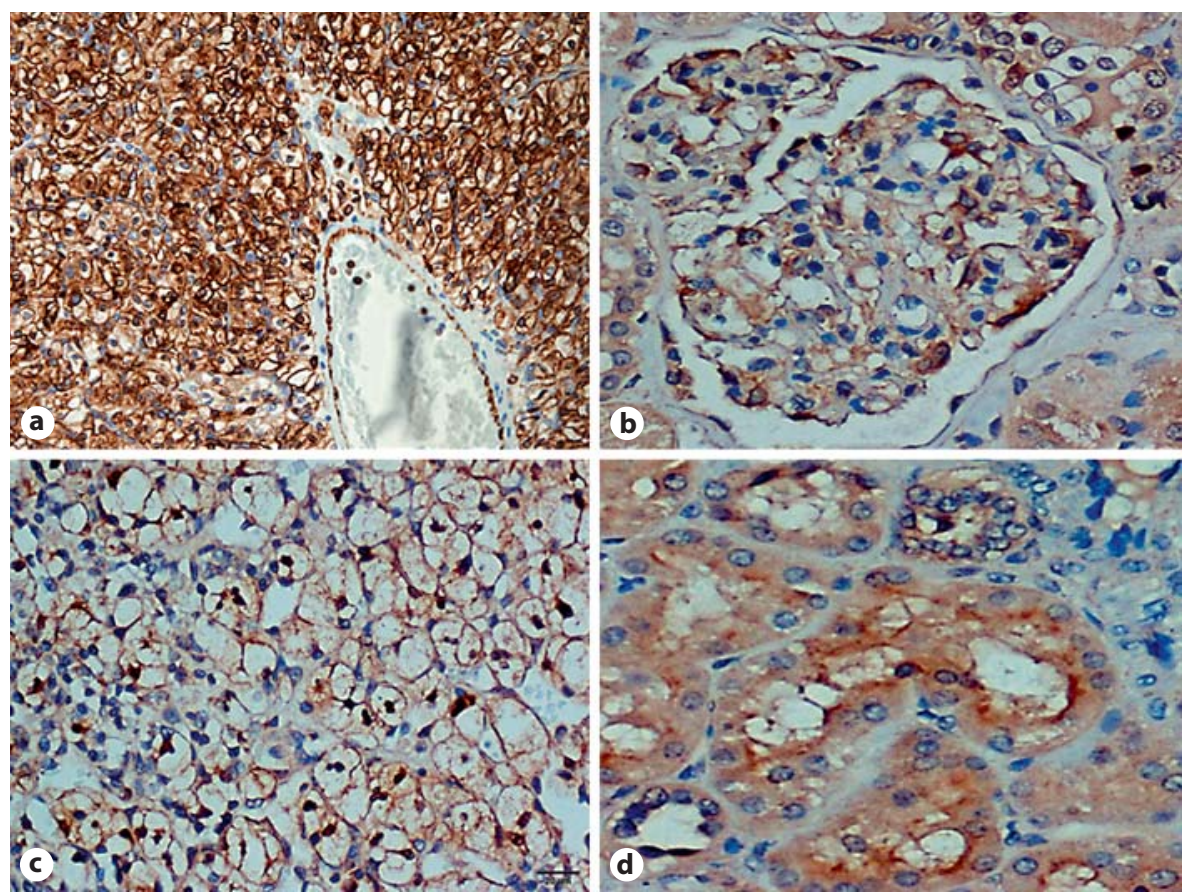

\section{Discussion}

Blanco et al. [2] reported a case of bilateral conventional RCC in a patient with chronic renal failure, diagnosed as FD only after the histological examination of the resected kidney. Cassiman et al. [1] described a bilateral multifocal RCC (papillary and clear cell type) in a kidney without the typical morphological alterations of FD. Both patients were males. We had the exceptional possibility to study a female patient with a first renal biopsy diagnosed as FD and a second specimen from nephrectomy that we could consider as 'repeat' biopsy. Moreover, no ERT was performed during the period between the two histological examinations, so we had the opportunity to study the natural history of FD. Foamy podocytes were present in the first renal biopsy, while in the second specimen similar vacuoles were easily detected also in the tubular cells. Focal segmental sclerosis was already present at the time of diagnosis; 6 years later, this finding was even more evident, probably because of the chronic evolution of untreated FD. The arteriole walls were a little more thickened in the second specimen, while we did not detect a severe vascular injury. The ultrastructural findings of our study were the most interesting features. In the first biopsy, inclusions were easily recognizable in epithelial cells. No alteration was evident in the endothelium. Instead, endothelial inclusions in nephrectomy were tiny, but frequent in glomeruli, interstitium and around the tumor. So, the first 'take home message' was that despite a normal renal function the kidney damage had a significant progressive evolution that could justify repeat biopsy as an important clinical instrument for patient management [3]. The second key point of our report was the possible pathogenetic relationship between FD and RCC. Were the zebra bodies in RCC cells only an epiphenomenon of a constitutive enzymatic deficit or, alternatively, a promoting factor involved in oncogenesis? Supporting this second hypothesis, many types of glycosphingolipids and gangliosides were found in a variety of renal non-neoplastic diseases (polycystic kidney disease, acute kidney injury, glomerulonephritis and diabetic nephropathy) and in RCC cells [5]. In fact, glycosphingolipids are thought to promote cell proliferation and, thus, tumor growth [6]. In vitro models had just shown that Gb3 are related to oxidative stress damage in a dose-dependent manner [7]. Interestingly, we know that the most powerful trigger in RCC development is oxidative damage (through Von Hippel-Lindau/hypoxia-inducible factor $1 \alpha-$ VHL/HIF pathway) [8]. In vivo models are still unavailable, but a preliminary investigation in FD patients under ERT found that elevated Gb3 levels are strongly related to decreased antioxidant defenses and increased levels of inflammatory cytokines (interleukin-6 and tumor necrosis factor- $\alpha$ ) [9]. Moreover, Lyso-Gb3 
was recently shown to increase transforming growth factor- $\beta_{1}$ production and CD74 expression in cultured renal cells. Lyso-Gb3 is globotriaosylsphingosine, a glycolipid accumulated in FD that is more water soluble than $\mathrm{Gb} 3$ [10]. Accumulating evidence has found that CD74 is upregulated in various kinds of cancers, implying that CD74 may play an important role in tumorigenesis. Genomic studies and immunohistochemical analyses suggest that CD74 is involved in tumor growth and metastasis [11]. The CD74 ligand (MIF) has also been proposed to play a role in renal cancer, regulated by the VHL/HIF pathway and overexpressed because of the loss of VHL [4]. So, we tested CD74 and MIF immunohistochemical expression in our nephrectomy specimen confirming their upregulation especially in tumor cells. We suggest a physiopathological link between $\mathrm{Gb} 3$ accumulation, oxidative stress and oncogenesis (VHL/HIF pathway) according to the hypothesis that high levels of MIF, secreted by RCC cells, activate a positive signaling cascade through the engagement of the cell surface receptors CD74. Although we present a single case, the possible correlation between $\mathrm{RCC}$ and FD is further sustained by the early onset of this tumor (50 years; median age for RCC 60-70 years) [12] and its rapid growth. As already proposed [13], only perspective studies will be able to clarify if FD patients have an incremental oncologic risk. Considering the bilateral presentation of the previous FD-related RCC in the literature, we will monitor the patient with a strong and seriated follow-up.

\section{Acknowledgements}

Special thanks to Antonella Tosoni, technician (University of Milan, Sacco Hospital) for her wonderful contribution to the ultrastructural analysis, and to Serena Buscone, BD (University Milan Bicocca), and Mattia Bugatti, technician (University of Brescia), for their expertise. Thanks to Dr. Marco Grasso, MD (San Gerardo Hospital, Monza), for the surgical management of the patient.

\section{Disclosure Statement}

The authors declare no competing interest or conflict of interest.

\section{References}

1 Cassiman D, Claes K, Lerut E, et al: Bilateral renal cell carcinoma development in longterm Fabry disease. J Inherit Metab Dis 2007; 30:830-831

-2 Blanco J, Herrero J, Arias LF, Garcia-Miralles N, Gamez C, Barrientos A: Renal variant of Anderson-Fabry disease and bilateral renal cell carcinoma. Pathol Res Pract 2005; 200:857-860.

-3 Hirashio S, Taguchi T, Naito T, et al: Renal histology before and after effective enzyme replacement therapy in a patient with classical Fabry's disease. Clin Nephrol 2009;71: 550-556.

4 Du W, Wright BM, Li X, et al: Tumor-derived macrophage migration inhibitory factor promotes an autocrine loop that enhances renal cell carcinoma. Oncogene DOI: 10.1038/onc.2012.143.
5 Cowart LA: Sphingolipids and Metabolic Disease. New York, Springer, 2011.

6 Chatterjee S, Pandey A: The Yin and Yang of lactosylceramide metabolism: implications in cell function. Biochim Biophys Acta 2008; 1780:370-382

7 Shen JS, Meng XL, Moore DF, et al: Globotriaosylceramide induces oxidative stress and up-regulates cell adhesion molecule expression in Fabry disease endothelial cells. Mol Genet Metab 2008;95:163-168.

8 Jonasch E, Futreal PA, Davis IJ, et al: State of the science: an update on renal cell carcinoma. Mol Cancer Res 2012;10:859-880.

$\checkmark 9$ Biancini GB, Vanzin CS, Rodrigues DB, et al: Globotriaosylceramide is correlated with oxidative stress and inflammation in Fabry patients treated with enzyme replacement therapy. Biochim Byophis Acta 2012;1822 226-232.
10 Sanchez-Niño MD, Sanz AB, Carrasco S, et al: Globotriaosylsphingosine actions on human glomerular podocytes: implications for Fabry nephropathy. Nephrol Dial Transplant 2011;26:1797-1802.

11 Liu YH, Lin CY, Lin WC, Tang SW, Lai MK, Lin JY: Up-regulation of vascular endothelial growth factor-D expression in clear cell renal cell carcinoma by CD74: a critical role in cancer cell tumorigenesis. J Immunol 2008;181:6584-6594.

12 Chang AE, Hayes DF, Pass HI, et al: Oncology: An Evidence-Based Approach. New York, Springer, 2007.

13 Cybulla M, Kleber M, Walter KN, et al: Is Fabry disease associated with leukaemia? Br J Haematol 2006;135:264-265. 